DisCRETE ANALYSIS, 2017:14, 5 pp.

www.discreteanalysisjournal.com

\title{
Sumsets as Unions of Sumsets of Subsets
}

\author{
Jordan S. Ellenberg \\ Received 22 May 2017; Published 6 September 2017
}

\begin{abstract}
We show that, for any subsets $S$ and $T$ of $\mathbb{F}_{q}^{n}$, there are subsets $S^{\prime} \subset S$ and $T^{\prime} \subset T$ such that $\left|S^{\prime}\right|+\left|T^{\prime}\right|<c_{q}^{n}$ for some $c_{q}<q$, and $\left(S^{\prime}+T\right) \cup\left(S+T^{\prime}\right)=S+T$.
\end{abstract}

The novel approach to additive combinatorics in abelian groups introduced by Croot, Lev, and Pach in [4] has led to rapid progress in a range of problems in extremal combinatorics: for instance, a new upper bound for the cap set probem [5], bounds for complexity of matrix-multiplication methods based on elementary abelian groups [2], bounds for the Erdős-Szemeredi sunflower conjecture [9], and polynomial bounds for the arithmetic triangle removal lemma [6]. In many of the applications, the original bound on cap sets in [5] does not suffice for applications: for instance, in [2] and [6] one needs to bound the size of a multi-colored sum-free set, a somewhat more general object.

In the present note, we use the Croot-Lev-Pach lemma, combined with an older result of Meshulam on linear spaces of low-rank matrices, to prove a still more general lemma on sumsets which implies many of the combinatorial bounds used in applications so far. Loosely speaking, we show that the sumset $S+T$ of two large subsets $S$ and $T$ of $\mathbb{F}_{q}^{n}$ can be expressed "more efficiently" as a union of sumsets of smaller subsets.

We first introduce some notation. Write $m_{d}$ for the number of monomials in $x_{1}, \ldots, x_{n}$ with degree at most $(q-1)$ in each variable and total degree at most $(q-1) n / 3$, and write $M\left(\mathbb{F}_{q}^{n}\right)$ for the upper bound proved in [5] for the size of a subset of $\mathbb{F}_{q}^{n}$ with no three-term arithmetic progressions; to be precise, we have

$$
M\left(\mathbb{F}_{q}^{n}\right)=3 m_{(q-1) n / 3}
$$

and $M\left(\mathbb{F}_{q}^{n}\right)$ is bounded above by $c^{n}$ for some $c<q$. (We note that for the sake of the present argument there is no need to consider prime powers $q$ other than primes.)

Theorem 1. Let $\mathbb{F}_{q}$ be a finite field and let $S, T$ be subsets of $\mathbb{F}_{q}^{n}$. Then there is a subset $S^{\prime}$ of $S$ and a subset $T^{\prime}$ of $T$ such that 
- $\left|S^{\prime}\right|+\left|T^{\prime}\right| \leq M\left(\mathbb{F}_{q}^{n}\right)$;

- $\left(S^{\prime}+T\right) \cup\left(S+T^{\prime}\right)=S+T$.

Applying Theorem 1 to the symmetric case $S=T$, we obtain the following corollary:

Corollary 2. Let $S$ be a subset of $\mathbb{F}_{q}^{n}$. Then $S$ has a subset $S^{\prime}$ of size at most $M\left(\mathbb{F}_{q}^{n}\right)$ such that $S^{\prime}+S=S+S$.

Proof. By Theorem 1 there are subsets $S_{1}$ and $S_{2}$ of $S$ such that $S+S=\left(S_{1}+S\right) \cup\left(S+S_{2}\right)$ and $\left|S_{1}\right|+\left|S_{2}\right| \leq$ $M\left(\mathbb{F}_{q}^{n}\right)$. Taking $S^{\prime}$ to be $S_{1} \cup S_{2}$ we are done.

This immediately implies the bound proved in [5] on subsets of $\mathbb{F}_{q}^{n}$ with no three terms in arithmetic progression:

Corollary 3 ([5]). A subset $S$ of $\mathbb{F}_{q}^{n}$ containing no three-term arithmetic progression has size at most $M\left(\mathbb{F}_{q}^{n}\right)$.

Proof. If $S$ has no 3-term arithmetic progression, then $S^{\prime}+S$ is strictly smaller than $S+S$ for every proper subset $S^{\prime} \subset S$ (because $S^{\prime}+S$ fails to contain $2 s$ if $s$ lies in the complement of $S^{\prime}$.) Thus, the subset $S^{\prime}$ guaranteed by Corollary 2 must be equal to $S$, whence $|S|=\left|S^{\prime}\right| \leq M\left(\mathbb{F}_{q}^{n}\right)$.

Theorem 1 also implies the bounds on multi-colored sum-free sets proved in [7] and [2]. (We note that [2] proves a substantially more general result which applies, for example, to arbitrary abelian groups of bounded exponent.)

Corollary 4 (Th 1, [7]). Let $S, T$ be subsets of $\mathbb{F}_{q}^{n}$ of the same cardinality $N$, assigned an ordering $s_{1}, \ldots s_{N}$ and $t_{1}, \ldots, t_{N}$ such that the equation $s_{i}+t_{i}=s_{j}+t_{k}$ holds only when $(j, k)=(i, i)$. Then $N \leq M\left(\mathbb{F}_{q}^{n}\right)$.

Proof. Let $S^{\prime}, T^{\prime}$ be chosen as in Theorem 1. Each sum $s_{i}+t_{i}$ therefore lies in either $S+T^{\prime}$ or $S^{\prime}+T$. But since $s_{i}+t_{i}$ cannot be expressed as $s_{j}+t_{k}$ for any other $j, k$, this implies that either $s_{i} \in S^{\prime}$ or $t_{i} \in T^{\prime}$. It follows that $N \leq\left|S^{\prime}\right|+\left|T^{\prime}\right| \leq M\left(\mathbb{F}_{q}^{n}\right)$.

We now prove Theorem 1. The proof is along the same lines as the arguments in the papers cited, but there is one new ingredient: a result of Meshulam [8] on linear spaces of matrices of low rank.

Proof. Let $V$ be the space of polynomials in $\mathbb{F}_{q}\left[x_{1}, \ldots, x_{n}\right]$ with degree at most $q-1$ in each variable and total degree at most $d$, that vanish on the complement of $S+T$. Then $\operatorname{dim} V$ is at least $m_{d}-q^{n}+|S+T|$. Write $\mathcal{M}$ for the space of of $|S| \times|T|$ matrices, where the rows are understood to be indexed by $S$ and the columns by $T$.

For each $P \in V$ we may consider the matrix $M(P) \in \mathcal{M}$ whose entries are $P(s+t)_{s \in S, t \in T}$. By the argument of the Croot-Lev-Pach lemma [4], this matrix has rank at most $2 m_{d / 2}$.

Note that $M$ is a homomorphism from $V$ to $\mathcal{M}$, which is injective: if $P$ lies in the kernel, it vanishes at $S+T$, but $P$ vanishes on the complement of $S+T$, so $P$ vanishes on every point of $\mathbb{F}_{q}^{n}$ and is 0 .

We thus can, and shall, think of $V$ as a vector subspace of $\mathcal{M}$ of dimension at least $m_{d}-q^{n}+|S+T|$, each of whose members has rank at most $2 m_{d / 2}$. 


\section{SUMSETS AS Unions OF SUMSETS OF SUbSETS}

The arguments of [5],[7],[2] proceed by showing that, if $S, T$ satisfy the conditions Corollary 4, then $V$ contains a diagonal matrix with at least $m_{d}-q^{n}+|S|$ nonzero entries, which implies

$$
m_{d}-q^{n}+|S| \leq 2 m_{d / 2},
$$

an upper bound on $|S|$. The mild novelty of the present paper is to exploit the Croot-Lev-Pach rank bound for the whole space $V$, not just for its subspace of diagonal matrices. The earlier papers use the easy fact that a vector space of diagonal matrices of dimension at least $r$ contains a matrix of rank at least $r$. For spaces of general matrices, the problem of controlling the maximal rank attained in a linear space of matrices is much richer. We will use a theorem of Meshulam [8, Theorem 1] in this area, which (rather surprisingly to us) turns out to be perfectly adapted to the combinatorial application. (Indeed, we did not set out to prove Theorem 1; rather, we encountered Meshulam's theorem and simply worked out what it had to say about sumsets when combined with the argument of [5].)

In the interest of self-containedness, we state Meshulam's theorem below.

Theorem 5 (Meshulam). Let $k$ be a field and $W$ a vector subspace of $M_{n}(k)$. For each $w \in W$ let $p(w) \in\{1, \ldots, n\} \times\{1, \ldots, n\}$ be the lexicographically first $(i, j)$ such that the entry $w_{i j}$ is nonzero, and let $\Sigma$ be the set of all $p(w)$ as $w$ ranges over $W$. Suppose every matrix in $W$ has rank at most $r$. Then there exists a set of $m$ rows and $m^{\prime}$ columns such that every element of $\Sigma$ is contained in one of the rows or one of the columns, and $m+m^{\prime} \leq r$.

We now return to the proof of Theorem 1. Choose an ordering on $S$ and an ordering on $T$. These choices endow the entries of a matrix in $\mathcal{M}$ with a lexicographic order. As above, for each matrix $A \in \mathcal{M}$, we denote by $p(A) \in S \times T$ the location of the lexicographically first nonzero entry of $A$.

We note that $p(M(P))$ cannot be an arbitrary element of $S \times T$, since $M(P)$ has equal entries at $(s, t)$ and $\left(s^{\prime}, t^{\prime}\right)$ whenever $s+t=s^{\prime}+t^{\prime}$. In particular, this means that $(s, t)$ and $\left(s^{\prime}, t^{\prime}\right)$ cannot both be $p(M(P))$ for polynomials $P \in V$; only the lexicographically prior of these two pairs can appear.

By Gaussian elimination, there is a basis $A_{1}, \ldots, A_{\operatorname{dim} V}$ for $V$ such that $p\left(A_{1}\right), \ldots, p\left(A_{\operatorname{dim} V}\right)$ are distinct. Now apply Theorem 5, which shows that there is a set of $2 m_{d / 2}$ lines (a line being a row or a column) whose union contains $p\left(A_{i}\right)$ for all $i$.

This set of lines consists of a subset of $S$, which we call $S_{0}$, and a subset of $T$, which we call $T_{0}$, satisfying $\left|S_{0}\right|+\left|T_{0}\right|=2 m_{d / 2}$.

We now have, for $i=1, \ldots, \operatorname{dim} V$,

$$
p\left(A_{i}\right)=\left(s_{i}, t_{i}\right)
$$

with either $s_{i} \in S_{0}$ or $t_{i} \in T_{0}$. What's more, $s_{i}+t_{i}$ and $s_{j}+t_{j}$ are distinct whenever $i$ and $j$ are. So the union of $S_{0}+T$ with $S+T_{0}$ contains at least $\operatorname{dim} V$ elements of $S+T$.

Since $\operatorname{dim} V \geq m_{d}-q_{n}+|S+T|$, the set $W$ of elements of $S+T$ not contained in $\left(S_{0}+T\right) \cup\left(S+T_{0}\right)$ has cardinality at most $q_{n}-m_{d}$. Let $S_{1}$ be a subset of $S$ of size $q_{n}-m_{d}$ such that each $w \in W$ is represented as $s+t$ for some $s \in S_{1}$. Then taking $S^{\prime}=S_{0} \cup S_{1}$ and $T^{\prime}=T_{0}$, we have that $S^{\prime}+T \cup S+T^{\prime}$ contains all of $S+T$; moreover,

$$
\left|S^{\prime}\right|+\left|T^{\prime}\right| \leq 2 m_{d / 2}+q^{n}-m_{d}
$$

and minimizing over $d$ we get the desired result. 
Remark 6. We note that the algebraic approach to bounding sumsets is much older than [4] and [5]; one ancestor, for instance, is Alon's short proof of the Erdôs-Heilbronn conjecture via combinatorial Nullstellensatz [1, Prop 4.2], which also proceeds by considering algebraic properties of a polynomial vanishing on the set of distinct sums in an abelian group (in that case a cyclic group.)

Question 7. Corollary 4, the bound on multi-colored sum-free sets, can be expressed in a more symmetric, and thus more appealing, form: Suppose $S, T, U$ are subsets of $\mathbb{F}_{q}^{n}$ such that the set

$$
\{(s, t, u) \in S \times T \times U: s+t+u=0\}
$$

forms a perfect matching between the three sets. Then $|S|=|T|=|U|$ is at most $M\left(\mathbb{F}_{q}^{3}\right)$. The proof, too, has a symmetric formulation; Tao introduced the notion of slice rank for tensors in $\mathbb{F}_{q}^{n} \otimes \mathbb{F}_{q}^{n} \otimes \mathbb{F}_{q}^{n}$, which was quickly generalized in many directions and applied to a range of further combinatorial problems (see e.g. [10].)

Symmetric methods of this type seem to be the most elegant way to approach these problems. Is there a way to state Theorem 1, and prove it, as a statement about solutions to $s+t+u=0$ which places the three summands on an equal footing?

Question 8. One naturally wonders whether Theorem 1 has an analogue for cyclic groups. That is: let $g(N)$ be the smallest integer such that, for any subsets $S$ and $T$ of $\mathbb{Z} / N \mathbb{Z}$, there are always $S^{\prime} \subset S$ and $T^{\prime} \subset T$ with $\left(S+T^{\prime}\right) \cup\left(S^{\prime}+T\right)=S+T$ and $\left|S^{\prime}\right|+\left|T^{\prime}\right| \leq g(N)$. What can we say about the growth of $g(N)$ ? Behrend's example [3] of a large subset of $\mathbb{Z} / N \mathbb{Z}$ with no three-term arithmetic progressions shows that $g(N)$ would have to be at least $N^{1-\varepsilon}$. Jacob Fox and Will Sawin explained to me that $g(N)=o(N)$ follows from known bounds for arithmetic triangle removal.

\section{Acknowledgments}

The author is supported by NSF Grant DMS-1402620 and a Guggenheim Fellowship. He thanks Jacob Fox, Will Sawin, the referees, and the readers of Quomodocumque for useful discussions about the subject of this paper.

\section{References}

[1] Noga Alon, Combinatorial nullstellensatz, Combinatorics, Probability and Computing 8 (1999), no. 1-2, 7-29. $\uparrow 4$

[2] Jonah Blasiak, Thomas Church, Henry Cohn, Joshua A Grochow, Eric Naslund, William F. Sawin, and Chris Umans, On cap sets and the group-theoretic approach to matrix multiplication, Discrete Analysis 2017:3. $\uparrow 1,2,3$

[3] Felix A Behrend, On sets of integers which contain no three terms in arithmetical progression, Proceedings of the National Academy of Sciences 32 (1946), no. 12, 331-332. $\uparrow 4$

[4] Ernie Croot, Vsevolod Lev, and P'eter Pál Pach, Progression-free sets in $\mathbf{Z}_{4}^{n}$ are exponentially small, Ann. of Math. 185 (2017), no. 1, 331-337. $\uparrow 1,2,4$

[5] Jordan S. Ellenberg and Dion Gijswijt, On large subsets of $\mathbb{F}_{q}^{n}$ with no three-term arithmetic progression, Ann. of Math. 185 (2017), no. 1, 339-343. $\uparrow 1,2,3,4$

[6] Jacob Fox and László Miklós Lovász, A tight bound for Green's boolean removal lemma, arXiv preprint arXiv:1606.01230 (2016). $\uparrow 1$ 


\section{SuMSETS AS Unions OF SUMSETS OF SUbSETS}

[7] Robert Kleinberg, A nearly tight upper bound on tri-colored sum-free sets in characteristic 2, arXiv preprint arXiv:1605.08416 (2016). $\uparrow 2,3$

[8] Roy Meshulam, On the maximal rank in a subspace of matrices, The Quarterly Journal of Mathematics 36 (1985), no. 2 , 225-229. $\uparrow 2,3$

[9] Eric Naslund and William F. Sawin, Upper bounds for sunflower-free sets, arXiv preprint arXiv:1606.09575 (2016). $\uparrow 1$

[10] Eric Naslund, The multi-slice rank method and polynomial bounds for orthogonal systems in $\mathbf{F}_{q}^{n}$, arXiv preprint arXiv:1701.04475 (2017). $\uparrow 4$

\section{AUTHOR}

Jordan S. Ellenberg

Professor

University of Wisconsin-Madison

ellenber [at] math [dot] wisc [dot] edu

http://www.math.wisc.edu/ ellenber 